\title{
An investigation into the satiating effects of differing quantities of protein consumed at breakfast.
}

\author{
E. Tolan and S. Drummond \\ Queen Margaret University, Musselburgh, Edinburgh EH21 6UU, UK
}

The fundamental contributing factor to the current obesity epidemic is energy imbalance. Satiety is one component that affects energy balance by influencing appetite and subsequent food intake. Highly satiating foods are therefore promising strategies to incorporate in the prevention or treatment of obesity. Evidence suggests that protein is the most satiating macronutrient ${ }^{(1)}$ and that higher protein intakes cause a decreased subsequent energy intake ${ }^{(2)}$. More specifically, consuming protein at breakfast has shown to initiate and sustain feelings of fullness for a longer period compared to when it is consumed at lunch or dinner ${ }^{(3)}$.

This study aimed to investigate if normal protein (NP; $15 \%$ energy), moderate protein (MP; $25 \%$ energy) and high protein (HP; $35 \%$ energy) quantities at breakfast proportionately increase subjective satiety and decrease within day energy intake.

In a single blind three way crossover study, 12 healthy subjects ( 4 male, 8 female) $(\mathrm{BMI}=23.9 \pm 0.65 \mathrm{~kg} / \mathrm{m} 2)$ aged $21-31$ years consumed three isocaloric breakfasts containing approximately $15 \%, 25 \%$ and $35 \%$ energy from protein, one week apart. Appetite parameters were measured via Visual Analogue Scale ratings before and after each breakfast and hourly thereafter. Dietary intake was recorded at baseline and on each test day via 24-hour diet diaries. Mean energy intake on test days was compared to habitual intake to assess each breakfasts effect on appetite and within day energy intake.

\begin{tabular}{|c|c|c|c|c|c|c|c|c|}
\hline \multirow[b]{2}{*}{ Dietary Intakes } & \multicolumn{2}{|c|}{ Baseline } & \multicolumn{2}{|c|}{ Normal Protein } & \multicolumn{2}{|c|}{ Medium Protein } & \multicolumn{2}{|c|}{ High Protein } \\
\hline & Mean & $\overline{\text { SEM }}$ & Mean & $\overline{\text { SEM }}$ & $\overline{\text { Mean }}$ & $\overline{\text { SEM }}$ & $\overline{\text { Mean }}$ & $\overline{\text { SEM }}$ \\
\hline Kcal & 2225 & 132 & 2037 & 130 & $1813^{*}$ & 133 & $1753^{*}$ & 230 \\
\hline MJ & $9 \cdot 31$ & 0.55 & 8.52 & $0 \cdot 54$ & $7 \cdot 59^{*}$ & 0.56 & $7 \cdot 34^{*}$ & 0.96 \\
\hline Protein (g) & $104 \cdot 9$ & 11.9 & $101 \cdot 5$ & $11 \cdot 8$ & $112 \cdot 6$ & $15 \cdot 2$ & $122 \cdot 5 * *$ & $12 \cdot 8$ \\
\hline $\mathrm{CHO}(\mathrm{g})$ & $278 \cdot 8$ & $17 \cdot 1$ & $243 \cdot 8$ & $16 \cdot 9$ & $233 \cdot 2$ & $13 \cdot 9$ & $205 \cdot 2 *$ & $10 \cdot 6$ \\
\hline Fat $(\mathrm{g})$ & $82 \cdot 3$ & $6 \cdot 2$ & 79.9 & $7 \cdot 9$ & $52 \cdot 7 *$ & $6 \cdot 5$ & $52 \cdot 9^{*}$ & $4 \cdot 7$ \\
\hline Total VAS score & & & & & & & & \\
\hline Fullness & & & $56 \cdot 2$ & $4 \cdot 8$ & 63.9 & $2 \cdot 8$ & $69 \cdot 2^{\# \#}$ & 1.8 \\
\hline Hunger & & & $38 \cdot 8$ & $3 \cdot 8$ & $30 \cdot 1$ & $2 \cdot 8$ & $29 \cdot 6^{\#}$ & 1.8 \\
\hline Desire to eat & & & $40 \cdot 9$ & $4 \cdot 4$ & 31.4 & 3.8 & $29 \cdot 7^{\#}$ & $2 \cdot 3$ \\
\hline
\end{tabular}

* Significantly different from Baseline;

** Significantly different from Baseline and NP;

\# Significantly different from NP;

\#\# Significantly different from NP \& MP; (RMANOVA followed by Post Hoc Bonferroni correction): $P<0 \cdot 05$.

Significant increases in fullness over 8 hours and significant decreases in hunger, capacity and desire to eat were observed post consumption of the HP breakfast compared to the NP breakfast. However, significant effects on food intake only occurred between the MP breakfast and baseline and the HP breakfast and baseline $(p<0.05)$ and not between breakfasts $(\mathrm{p}>0.05)$, although an emerging trend in mean energy intake differences between breakfast conditions was evident. If mean energy intake reductions were to persist in the long term, this may prove to be a beneficial strategy within the multidisciplinary prevention or treatment of obesity. Therefore, longer term studies are required to elucidate such findings.

1. Poppitt SD, McCormack D \& Buffenstein R (1998) Physiol Behav 64 (3), 279-285.

2. Westerterp-Plantenga MS, Lejeune MP, Nijs I et al. (2004) Int J Obes Rel Met Dis 28(1), 57-64.

3. Leidy HJ, Bossingham M.J, Mattes RD et al. (2009) Brit J Nut 101(6), 798-803. 